\title{
Reingreso de mujeres en el primer segmento de la educación de jóvenes y personas adultas en Porto Velho, Rondônia
}

\author{
The Return of Women to the First Segment of School Education for Youth and Adults in \\ Porto Velho, Rondônia
}

O (re) ingresso de mulheres no primeiro segmento da Educação de Jovens e Adultos em Porto Velho, Rondônia

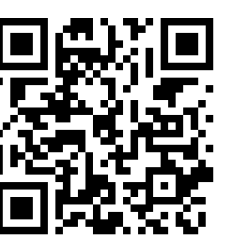

Pontifícia Universidad Catolica do Rio Grande do Sul

Rondônia, Brasil

karyfalcao@yahoo.com.br

iD http://orcid.org/0000-0001-5684-8906

Adriano Campos-Prates

Faculdade de Ciências Administrativas y Tecnologia

Rondônia, Brasil

campos.sb12@gmail.com

http://orcid.org/0000-0003-3840-3262

Recibido • Received • Recebido: 15 / 08 / 2017

Corregido • Revised • Revisado: 17 / 09 / 2018

Aceptado • Accepted • Aprovado: 22 / 02 / 2019

\begin{abstract}
Resumen: El objetivo de este estudio es presentar los desafíos y perspectivas en el retorno de las mujeres a la escuela en el primer segmento de la educación de jóvenes y personas adultas (EJA), ofrecida en el nocturno en las escuelas de la red municipal de enseñanza. La investigación fue desarrollada en cuatro escuelas públicas de Porto Velho, ubicadas en diferentes zonas de la ciudad con 48 mujeres entre 15 y 65 años de edad. El estudio evidenció que el alejamiento de estas mujeres de la escuela permanece relacionado con factores familiares como la prohibición de estudiar por el marido, la preocupación por la creación y el cuidado de su progenie y el quehacer del hogar. En cuanto al retorno de estas mujeres a la escuela, las principales dificultades encontradas en el estudio fueron a la preocupación por los hijos y las hijas en cuanto a la implicación con drogas y la criminalidad, el cansancio del trabajo, la edad avanzada y la falta de seguridad en los barrios de la ciudad.
\end{abstract}

Palabras claves: Mujeres; estudiantes; trabajo; escuela; jóvenes y personas adultas. 
doi: http://dx.doi.org/10.15359/ree.23-2.3

URL: http://www.una.ac.cr/educare

CORREO: educare@una.cr

\begin{abstract}
The objective of this study is to present challenges and perspectives on the return of women to the first segment of School Education for Youth and Adults (EJA), offered in night schools of the municipal school network. The research was conducted with 48 women, between 15 and 65 years of age, in four public schools of Porto Velho, located in different areas of the city. The study showed that factors preventing these women from going to school remain related to family factors such as their husbands' prohibition of studying, concerns about the raising and care of their children, and household chores. As for the return of these women to school, the main difficulties encountered in the study were the concern about their children in relation with drug involvement and crime, work fatigue, old age and lack of security in the city's neighborhoods.
\end{abstract}

Keywords: Women; students;job; School; youth and adults.

Resumo: $O$ objetivo deste estudo é apresentar os desafios e perspectivas no retorno de mulheres à escola no primeiro segmento da Educação de Jovens e Adultos - EJA, ofertada no noturno das escolas da rede municipal de ensino. A pesquisa foi desenvolvida em quatro escolas públicas de Porto Velho, localizadas em diferentes zonas da cidade com 48 mulheres entre 15 e 65 anos de idade. $O$ estudo evidenciou que $o$ afastamento destas mulheres da escola permanece relacionado a fatores familiares como a proibição de estudar pelo marido, a preocupação com a criação e o cuidado com os filhos e os afazeres do lar. Quanto ao retorno destas mulheres à escola, as principais dificuldades encontradas no estudo foram à preocupação com os filhos quanto ao envolvimento com drogas e a criminalidade, o cansaço do trabalho, a idade avançada e a falta de segurança nos bairros da cidade.

Palavras-chave: Mulheres; estudantes; trabalho; escola; jovens e adultos.

\title{
Introducción
}

La educación de jóvenes y personas adultas, conforme con la Ley de Directrices y Bases de la Educación Nacional (LDB, Ley 9.394 / 96) (Congreso Nacional, Brazil, 1996), está dirigida al estudiantado que no tuvo oportunidad de asistir a la escuela en tiempo propio. Sin embargo, el retorno de este estudiantado a la escuela está marcado por una serie de dificultades que acompañan este proceso de reingreso.

La rutina de trabajo con el retorno a la escuela asociada a la edad avanzada y las preocupaciones con la familia y otras situaciones del trabajo, además de contribuir al abandono escolar, es otro elemento que debe ser pensado por el personal educativo que actúa en la educación de jóvenes y personas adultas.

Este estudio tiene por objetivo presentar las dificultades y las perspectivas de mujeres entre 15 y 65 años de edad, con el retorno a la escuela, en el período nocturno, en las clases del primer segmento de la EJA. La red municipal de enseñanza ofrece el primer segmento de la EJA en 14 escuelas en el Municipio de Porto Velho, la encuesta tuvo lugar en 04 (cuatro) escuelas ubicadas en las cuatro zonas de la ciudad y tuvo como participantes a 48 mujeres. 
Entre las posibilidades teóricas del estudio de género, se optó por investigar sobre el reingreso de mujeres en la EJA específicamente por pensar en el trabajo femenino aliado a las cuestiones sociales e históricas atribuidas a los fenómenos derivados de la cultura machista fijada a partir de relaciones construidas.

De esta forma, este artículo buscó la referencia teórica del estudio de Siqueira (2009), que investigó sobre el retorno de jóvenes y personas adultas a los estudios entre 20 y 40 años de edad y las implicaciones con el mundo del trabajo, las preocupaciones con el futuro, tiempo perdido y las relaciones interpersonales. Para contextualizar la educación de personas jóvenes y adultas, Moura (2003) apunta a las primeras manifestaciones en educación en Brasil como un modelo orientado hacia jóvenes y personas adultas inicialmente, en el sentido de catequización y alfabetización por los grupos jesuitas.

Se estudiaron dos investigaciones en este proceso de comprensión histórica de la educación de la población escogida, el de Strelhow (2010) y el de Friedrich, Benite, Benite y Pereira (2010) que tratan del posicionamiento del profesorado y la formación docente para actuar con la EJA.

En cuanto al retorno de las mujeres a la escuela, Eiterer, Dias y Coura (2014) presentan los juicios anticuados de la mujer para justificar la exclusión de estas mismas tanto del mercado de trabajo como de los procesos de escolarización. En cuanto a los resultados de la investigación, el estudio de Andrade (2008) atribuye el abandono de la mujer a la escuela a problemas estrictamente relacionados con la maternidad, también se evidencia, en este estudio, que los factores de seguridad y las cuestiones históricas de género aparecen con mucha frecuencia en las encuestas de las mujeres estudiantes que reingresaron al primer segmento EJA.

\section{Educación de personas jóvenes y adultas: Contextualización histórica}

En la educación brasileña, a partir del período colonial, se puede percibir, en principio, una mirada dirigida a la atención de la niñez. Sin embargo, los grupos indígenas adultos también fueron resignados a una intensa acción educativa con propósitos de catequización y alfabetización, y para contribuir con el trabajo de la mano de obra esclava en la labranza y en las actividades extractivistas. La catequesis y la alfabetización de indígenas, como una de las atribuciones de la Compañía de Jesús, era responsable de la iniciación en la fe católica y de facilitar la comunicación entre la población portuguesa e indígena con la inserción de la lengua portuguesa en su dialecto.

Hasta la expulsión de los jesuitas en 1759 por Marques de Pombal, la educación de sujetos adultos estaba orientada al desarrollo de la educación de la elite religiosa, que dirigía los intereses a personas adolescentes y adultas antes que a la niñez, por la necesidad de predominio del proselitismo católico (Moura, 2003). 
doi: http://dx.doi.org/10.15359/ree.23-2.3

URL: http://www.una.ac.cr/educare

CORREO: educare@una.cr

A pesar del gran número de colegios dejados por los jesuitas, la educación Pombalina no se preocupó por la educación de personas adultos. La educación de clases marca este período con características bien distintas entre la clase rural brasileña y los procesos de elitización de la clase dominante, lo cual se extiende hasta el período republicano. Sin embargo, podemos decir, conforme con Moura (2003), que la población adulta de las clases menos afluentes que tenían la intención de estudiar no encontraban oportunidades con la reforma de Marques de Pombal, porque la enseñanza era un privilegio de minorías y esta reforma pretendía dar atención prioritaria a la enseñanza superior.

La analogía de la educación brasileña fue, entonces, marcada por el elitismo que restringía la educación a las clases más pobres. Por lo tanto, para Strelhow (2010), la historia de la educación fue desmedida por una situación singular que era del conocimiento formal monopolizado por las clases dominantes, específicamente para los hijos de los grupos colonizadores. Con la Constitución Imperial surge la necesidad de significados más amplios a la educación, que garanticen a toda la ciudadanía la instrucción primaria, pero esta ley quedó solo en el papel.

A partir del acto constitucional de 1834, se responsabilizó a las provincias de la instrucción primaria y secundaria de todas las personas, pero específicamente designada a individuos adolescentes y adultos. Esta educación era orientada por principios misioneros y caritativos (Strelhow, 2010).

En este trabajo, se aborda el estudio de Strelhow (2010), quien a través de elementos históricos propone analizar la acción política que posibilitó lo que hoy podemos denominar de educación de jóvenes y personas adultas. En cuanto al analfabetismo, el autor los considera como niños o niñas, incapaces de pensar por sí. El estudio contextualiza la educación de este período imperial, pasando por los períodos republicanos, las marcas de los movimientos sociales en la implementación de políticas de educación, la represión de la dictadura militar hasta los días más actuales.

Con la excesiva presión internacional para la eliminación del analfabetismo en dichas "naciones atrasadas" este fue uno de los motivos para el aglomerado de la Primera Campaña Nacional de Alfabetización. Otro factor que contribuyó a una educación masiva fue la consideración de la persona analfabeta como ignorante, incapaz, cabeza dura, sin forma para las letras. Por lo tanto, las personas adultas aún no alfabetizadas deberían recibir la misma educación empleada en la educación infantil, pues eran personas adultas analfabetas inhabilitadas para comprender (Strelhow, 2010).

Otro estudio que hace un abordaje histórico de la escolarización de jóvenes y personas adultas en la trayectoria de la educación brasileña es el análisis de Friedrich et al. (2010) y el paralelo de la formación de profesorado para actuar con este estudiantado entre el período de 1973 a 2007. Es importante resaltar que en este período, en la Ley n.o 5.692 (Congreso Nacional, 
Brazil, 1971), este grado de enseñanza apunta a contemplar a la juventud adulta como propuesta de reposición de escolaridad, el aprovisionamiento como perfeccionamiento, pues en 1996, la nueva Ley de Directrices y Bases de la Educación Nacional trata la educación para quienes no tuvieron acceso en edad propia como educación de jóvenes y personas adultas, y de modo muy bien valorado. El estudio presenta las propuestas de redemocratización con el Movimiento Brasileño de Alfabetización (MOBRAL), su extinción, la Fundación EDUCAR y el Programa Nacional de Alfabetización y Ciudadanía (PNAC), creado por el gobierno Collor de Mello.

Sin embargo, la permanencia de alumnado adulto en el aula o el éxito en el desempeño académico no está vinculado solamente a los programas de acceso a la escolarización. De acuerdo con Siqueira (2009), se pueden constatar los motivos de la desistencia de los estudios, tiempo de alejamiento, la motivación del retorno a la escuela, entre otros aspectos. Es importante resaltar la importancia del motivo del retorno a la escuela, recordando que esta población, la mayoría de las veces, está constituida por grupos trabajadores: incentivo que reciben, valorización por la familia, autoestima, incentivos por los entes empleadores, por amistades, por colegas, años anteriores, etc.

Siqueira (2009) cuestiona el trabajo y las relaciones con las nuevas oportunidades con el mercado convencional, que para el individuo joven y adulto representa "arreglar un empleo mejor, un mejor trabajo, un trabajo fijo, conseguir un empleo, mantener el empleo" y muchas otras palabras de quien busca la EJA.

\section{La mujer trabajadora y el retorno la escuela}

De acuerdo con Eiterer et al. (2014), se evidencia la presencia de interdicción basada en un juicio anticuado en el que la mujer no necesita estudio y que va a la escuela para "perderse o buscar novio". A primera vista esta concepción parece cosa del pasado; sin embargo, es percibido todavía en los tiempos de hoy.

De acuerdo con los estudios de Siqueira (2009) en relación con el retorno de jóvenes y sujetos adultos a los estudios después de 20,30 y 40 años, el principal problema enfrentado por los mismos grupos etarios estaba ligado a la valorización de los estudios por estudiantes con familia ya formada. Estos sujetos adultos se preocupan por la descendencia, diciendo que quieren "dar el ejemplo a los hijos o a los nietos", creyendo que su actitud de volver a la escuela no es más que una forma de animar a sus familiares a seguir estudiando. Las relaciones familiares se fortalecen con este acto de decisión, pues los padres o madres-estudiantes influencian activamente a sus hijos e hijas-estudiantes para que también logren un buen desempeño escolar.

Siendo así, estos grupos de estudiantes anhelan mejores oportunidades para sí y para su familia, depositando sus esperanzas en la escuela. Aunque el estudio de Siqueira (2009) no está 
doi: http://dx.doi.org/10.15359/ree.23-2.3

URL: http://www.una.ac.cr/educare

CORREO: educare@una.cr

estrictamente vinculado al retorno de las mujeres, sus resultados demuestran que después de la recepción del Certificado de Conclusión de la Enseñanza Fundamental o Media, estos padres, madres-estudiantes proporcionan tanto para sí y para sus familiares una sensación de sentir satisfacción por haber logrado alcanzar objetivos que fueron tan difíciles de superar en su época.

Los estudios sobre EJA realizados por Siqueira (2009) revelan que la persona joven y la adulta estudiante no puede tratarse como si fuera un niño o niña cuya historia de vida comienza. Estos grupos de estudiantes quieren y necesitan visualizar la aplicación inmediata de lo que están aprendiendo. Al mismo tiempo, presentan temores, se sienten amenazados, pues su "ignorancia" trae tensión, angustia y complejo de inferioridad. A menudo tienen vergüenza de hablar de sí, de su casa, de la frustrada experiencia de su infancia en relación con la escuela.

Según Schwartz (2012), jóvenes y personas adultas, que vivieron sin dominar la lectura y la escritura en un mundo donde esta función es obligatoria, deben recibir una forma de educación orientada a despertar la conciencia de la importancia de alfabetizarse e instruirse: Los sujetos adultos analfabetos vivieron años circulando y trabajando en una cultura sin saber leer ni escribir. Entonces, es natural que tengan dudas sobre lo que es leer, qué es escribir y para qué sirven. ¿En qué puede ese aprendizaje contribuir cualitativamente a la vida? Estas cuestiones deben aclararse al principio, en el primer día, si es posible, en el contrato pedagógico. Pero no sirve aclarar lógicamente una cuestión y luego no "corporificar las palabras por el ejemplo" No sirve decir porque es bueno saber leer y escribir y cómo es malo no gustar ni leer ni escribir. Nadie necesita actitudes sin sentido ni significado sino con creencia, convicción y coherencia.

Andrade (2008), en un estudio que tuvo como objetivo discutir y problematizar las situaciones que posibilitar la exclusión de mujeres en la escuela, buscó analizar los aspectos volcados a la maternidad como principal elemento de distanciamiento de mujeres y escuela. Sin embargo, los conceptos arraigados de que el lugar de la mujer es en casa en los quehaceres domésticos y en el cuidado de los niños y niñas, en este proceso de escolarización negada y escolarización tardía, pueden ser el elemento indicador para el retorno y permanencia de estos grupos de estudiantes cuando la escuela ejerce su función democratizadora y de práctica de la ciudadanía aproximada con la perspectiva post-estructuralista.

Conforme Andrade (2008), es importante resaltar que: Si, antes el lugar de la mujer era en casa, para cuidar de los quehaceres domésticos y de la niñez, hoy el lugar de la mujer también es en la escuela, pero no con el objetivo primero, sino con el objetivo de promover la profesionalización femenina para competir con los hombres en el mercado de trabajo dentro de una mayor equidad.

Si la escuela es un espacio de construcción social y profesionalización, debe añadírsele, a su currículo, una serie de actividades que posibiliten la discusión de género y, en especial, revelar que la mujer posee las más variadas ambiciones, como la concreción del sueño de ser capaz de 
dar continuidad a los estudios. Una vez finalizada la enseñanza primaria y secundaria, cursar una graduación o curso técnico y también aprender más. En cuanto a la continuación de los estudios, las investigadoras Oro, Weschenfelder y Stecanela (2010) afirman que la elevación de la escolarización, así como la inserción de la mujer en el mercado de trabajo, trae a ellas nuevas perspectivas de mejoras de vida. Sin embargo, algunas mujeres se posicionaron de forma poco favorable en cuanto a esta cuestión, pues, tal vez, no todas se apropiaron de la importancia de una formación continuada, pues simplemente desean concluir los estudios por la necesidad inmediata de mantener su empleo actual, no preocupándose por retornos más significativos que podrían ser adquiridos a largo plazo si continuasen sus estudios.

La escolarización, así como la inclusión de la mujer en el mercado de trabajo, trae a estas nuevas perspectivas de futuro; sin embargo, es importante destacar que algunas mujeres entrevistadas se posicionaron de forma menos favorable en cuanto a esta cuestión, ya que, tal vez, no todas se han percatado de la importancia de una formación continuada, entonces, simplemente desean concluir los estudios por la necesidad inmediata de mantener su empleo actual, no preocupándose por futuros retornos más significativos que podrían ser adquiridos a largo plazo, con la continuación de sus estudios (Oro et al., 2010).

Hasta ese momento, de volver a estudiar, las mujeres enfrentan dificultades que recorren por el hecho de quedarse poco tiempo con los hijos o hijas, enfrentan cansancio físico de la jornada de trabajo, el descrédito de la familia, el miedo al reanudamiento, el difícil acceso de la escuela hasta su casa, su edad, así como la incompatibilidad de horarios entre el trabajo y la escuela. Muchas veces, esta dificultad de independencia que imposibilita su salida de casa para estudiar y trabajar deriva también de la ausencia de infraestructura para la atención social, como guarderías y escuelas. La mujer madre, esposa y trabajadora, siente la necesidad de ir a la búsqueda de la escolarización y cualificación profesional, aunque tardía, pero esto solo es posible cuando su progenie ha crecido y no depende tanto de cuidados maternos. Una de las mujeres entrevistadas afirma que a pesar de tener cuatro hijos y estar separada del marido, esto no es más una dificultad que pueda impedir la reanudación de los estudios (Oro et al., 2010).

Una visión positiva con respecto a la propuesta educativa de la EJA, así como a los resultados que proporciona en las vidas, es que se constituye en un espacio de socialización de la mujer: Recuperar el tiempo perdido con dedicación y perseverancia, ir a la búsqueda de un mejor futuro para ella y sus familias.

\section{Método}

Este estudio es una investigación empírica, de naturaleza cualitativa, aunque los datos fueron configurados y sometidos a cálculo porcentual con base en el número total de respuestas de las categorías. La etapa del análisis cualitativo comprendió los procesos de pre-análisis, exploración y 
doi: http://dx.doi.org/10.15359/ree.23-2.3

URL: http://www.una.ac.cr/educare

CORREO: educare@una.cr

tratamiento de las respuestas y su interpretación, conforme con los objetivos de la investigación. La organización de los contenidos fue abordada de acuerdo con el objetivo propuesto de realizar una revisión sobre el reingreso y las dificultades que acarrea (Günther, 2006).

\section{Participantes}

Participaron de esta encuesta 48 mujeres matriculadas en el primer segmento del curso de educación de jóvenes y personas adultas (EJA) de cuatro escuelas de la red municipal de enseñanza de la ciudad de Porto Velho (RO). El criterio de selección de las escuelas obedeció a la orden de localización en relación con las zonas de la ciudad.

El curso EJA de $1^{\circ}$ segmento es ofrecido en Porto Velho solamente por la red municipal de enseñanza distribuido en 14 escuelas en el período nocturno. Del total de estudiantes que se matricularon en el primer segmento del curso, 123 son mujeres según se especifica en la Tabla 1.

Tabla 1: Identificación de las Estudiantes participantes

\begin{tabular}{|c|c|c|c|c|c|c|c|c|c|c|}
\hline \multirow[t]{2}{*}{$\begin{array}{l}\text { Escuelas } \\
\text { Búsqueda }\end{array}$} & \multirow[t]{2}{*}{$\begin{array}{l}\text { Dirección/ } \\
\text { Zona }\end{array}$} & \multicolumn{4}{|c|}{$\begin{array}{l}\text { Total de } \\
\text { estudiantes EJA } \\
\text { 10 Segmento }\end{array}$} & \multicolumn{3}{|c|}{$\begin{array}{c}\text { Total } \\
\text { Estudiantes }\end{array}$} & \multicolumn{2}{|c|}{$\begin{array}{l}\text { Número } \\
\text { de mujeres } \\
\text { participantes }\end{array}$} \\
\hline & & $1^{a}$ & $2^{a}$ & $3^{a}$ & $4^{a}$ & Geral & Hom & Mul & Total & $\%$ \\
\hline $\begin{array}{l}\text { Escola Municipal de } \\
\text { Educação Infantil e } \\
\text { Fundamental - Nacional }\end{array}$ & $\begin{array}{l}\text { Rua: Wanderlei Pontes, } 3298 \\
\text { Bairro Nacional. Zona Oeste }\end{array}$ & 20 & 06 & 12 & 11 & 49 & 20 & 29 & 15 & 51,72 \\
\hline $\begin{array}{l}\text { Escola Municipal de Ensino } \\
\text { Fundamental - Padre } \\
\text { Chiquinho }\end{array}$ & $\begin{array}{l}\text { Avenida: Campos Sales, } 881 \\
\text { Bairro Areal } \\
\text { Zona Central. }\end{array}$ & 15 & 15 & 16 & 20 & 66 & 31 & 35 & 14 & 40,00 \\
\hline $\begin{array}{l}\text { Escola Municipal de Ensino } \\
\text { Fundamental - Joaquim } \\
\text { Vicente Rondon }\end{array}$ & $\begin{array}{l}\text { Rua: Garopaba, 2615, } \\
\text { BairroCohab-Zona Sul }\end{array}$ & 18 & 17 & 18 & 18 & 71 & 40 & 31 & 9 & 29,03 \\
\hline $\begin{array}{l}\text { Escola Municipal de Ensino } \\
\text { Fundamental - Ulisses } \\
\text { Soares Ferreira }\end{array}$ & $\begin{array}{l}\text { Rua: José Amador dos Reis, } \\
\text { n²938, } \\
\text { B. JK I. Zona Leste }\end{array}$ & - & 21 & 20 & 31 & 72 & 44 & 28 & 10 & 35,71 \\
\hline TOTAL & & 53 & 59 & 66 & 80 & 258 & 135 & 123 & 48 & 39,02 \\
\hline
\end{tabular}

Nota: Elaboración propia. 
En las cuatro escuelas participantes de la investigación, en total de 258 estudiantes que se matricularon en la EJA primer segmento en el período nocturno, el 39,02\% de las mujeres fueron sometidas a la entrevista. En todas las cuatro escuelas el equipo de investigación tuvo acceso al aula y las entrevistas se realizaron individualmente.

\section{Contexto de la investigación}

Las escuelas seleccionadas para la investigación fueron inicialmente visitadas por el equipo de investigación y programadas para la realización de las entrevistas. Estas fueron seleccionadas específicamente por estar ubicadas en cuatro puntos estratégicos, es decir, una escuela en cada zona de la ciudad.

El estudiantado del curso EJA en el primer segmento se caracterizan por estar mucho tiempo fuera de aula o porque nunca tuvieron acceso a los medios de escolarización. En todas las salas visitadas durante la investigación, se observó que el número de mujeres era muy reducido en relación con el total de estudiantes. Estos contextos están relacionados con la participación de las mujeres en el presupuesto familiar con una doble jornada de trabajo, así como otros elementos señaladores en los resultados de la investigación.

Es importante resaltar que el curso EJA para el primer segmento no es ofrecido en Porto Velho por las escuelas de la red estatal de enseñanza. Solo 14 escuelas de la red municipal ofrecen el curso destinado a estudiantes que no tuvieron acceso a la escolarización en edad propia.

\section{Instrumentos}

Para conocer las concepciones de las mujeres sobre la importancia de la escuela en la promoción del desarrollo social y cognitivo, así como para identificar los principales obstáculos al acceso y la permanencia de las mujeres en la escuela después de un largo período de alejamiento ocasionado por innumerables factores, se adoptó por una investigación descriptiva con enfoque cualitativo.

De esta forma, se utilizó una entrevista semiestructurada compuesta por dos ejes: un eje identificador del perfil de las participantes (edad, actuación profesional, tiempo dedicado al trabajo, escuela que estudia y serie / año); y cuatro cuestiones que permiten comprender la cantidad de tiempo en que estas mujeres se quedaron sin estudiar: los motivos que llevaron a abandonar la escuela en edad propia, las dificultades para volver a estudiar y sobre quién es el principal mantenedor de la familia y cuál es la principal participación de estas mujeres en el presupuesto familiar. 
doi: http://dx.doi.org/10.15359/ree.23-2.3

URL: http://www.una.ac.cr/educare

CORREO: educare@una.cr

Todas las escuelas fueron notificadas a través de un documento con los objetivos de la investigación y la presentación del equipo investigador. También se informó a la población estudiantil y se orientó en cuanto a la decisión de participar o no de la investigación.

En las cuatro escuelas, el 39,02\% de las mujeres aceptaron participar en las entrevistas, y el equipo investigador realizó dos intervenciones. La primera fue de una conversación con el equipo gestor de las escuelas para presentar el proyecto y programar las visitas a las aulas para seleccionar estudiantes. En la segunda visita, el equipo investigador entraba en sala con la autorización del profesorado responsable de las clases y seleccionaba a las participantes.

Es importante señalar que, durante las clases, se percibió un número muy grande de estudiantes ausentes. El día de las entrevistas, había salas con 20 estudiantes en las que estaban presentes solo 12. El profesorado fue unánime en informar que el público que frecuenta el curso tiene dificultades para frecuentar las clases regularmente. En su mayoría, son responsables de mantener financieramente a la familia y los horarios de trabajo acumulados, con el cansancio del día a día, dificultan aún más la permanencia en la escuela. Esta situación aumenta considerablemente en el caso de las mujeres, donde los factores se agregan con la responsabilidad de la educación y el cuidado de su progenie y los deberes de casa.

\section{Procedimientos}

En relación con los criterios éticos de investigación, se adoptó en este artículo por mantener el anonimato de las mujeres participantes. Durante la investigación, las participantes eran invitadas a responder individualmente a la entrevista semiestructurada aplicada y registrada textualmente. Para la recolección de los datos, las escuelas fueron comunicadas a través del Oficio N. 035 / PED / FATEC dirigido a cada dirección de la escuela para solicitar la autorización para la participación en la investigación, e informar de los objetivos de la investigación, su confidencialidad y el carácter voluntario.

Los datos fueron tratados a partir de contenido cualitativo, posteriormente fueron sometidos a cálculo porcentual con base en el número total de respuestas de las categorías. La etapa del análisis cualitativo comprendió los procesos de pre-análisis, exploración y tratamiento de las respuestas e interpretación de estas, conforme con los objetivos de la investigación. Las respuestas fueron transcritas integralmente, obedeciendo a todos los criterios de respetar el modo en que las entrevistadas presentaban sus opiniones. Así, tras la realización de todas las 48 entrevistas, se realizaron varias lecturas entre el equipo investigador del material construido a través de la entrevista y de las categorías construidas, organizadas según la frecuencia de respuestas semánticamente similares o divergentes, considerando los modos de representatividad, homogeneidad y pertinencia. 


\section{Resultados y discusión}

En cuanto al análisis de los datos recolectados, la investigación obedeció a los criterios fenomenológicos de descripción, teniendo como referencia la transcripción íntegra de las entrevistas. La reducción de las informaciones obedeció a procesos de síntesis, la interpretación como comprensión de los fenómenos y la organización de las unidades en las respuestas presentadas por las 48 participantes.

En cuanto a la edad y la profesión de las estudiantes entrevistadas, el $45,83 \%$ de ellas tiene edad entre 46 y 65 años y solamente seis mujeres tienen edad entre 15 a 25 años, según se especifica en la Tabla 2 y la Tabla 3.

Tabla 2: Edad de las estudiantes participantes

\begin{tabular}{ccc}
\hline Edad & Número de mujeres & $\%$ \\
\hline Entre 15 a 25 años & 06 & 12,51 \\
Entre 26 a 35 años & 10 & 20,83 \\
Entre 36 a 45 años & 10 & 20,83 \\
Entre 46 a 65 años & 22 & 45,83 \\
\hline TOTAL & 48 & 100 \\
\hline
\end{tabular}

Nota: Elaboración propia.

Tabla 3: Oficios de las estudiantes participantes

\begin{tabular}{lcc}
\hline Oficios de las participantes & Número de Mujeres & $\%$ \\
\hline Del hogar & 17 & 35,41 \\
Vendedora & 01 & 2,08 \\
Diaria/doméstica & 06 & 12,50 \\
Estudiante & 06 & 12,50 \\
Agricultora & 01 & 2,08 \\
Funcionaria publica & 01 & 2,08 \\
Cosmética & 02 & 4,16 \\
Cocinera & 01 & 2,08 \\
Servicios generales/veladora & 10 & 20,83 \\
Jubilación/penalización & 02 & 4,16 \\
No ha informado & 01 & 2,08 \\
\hline TOTAL & 48 & 100 \\
\hline
\end{tabular}

Nota: Elaboración propia. 
doi: http://dx.doi.org/10.15359/ree.23-2.3

URL: http://www.una.ac.cr/educare

CORREO: educare@una.cr

El estudiantado de las cuatro escuelas encuestadas representan un grupo de mujeres que desarrollan una jornada de trabajo y estudio considerado múltiple: trabajo, escuela, casa, hijos, hijas y otros. Solo 6 mujeres se identificaron exclusivamente como estudiantes en una proporción del $12,50 \%$. Las demás ejercen tareas que están asociadas a los motivos que las alejaron de la escuela en el momento oportuno o que las colocaron en situación de nunca haber frecuentado la escuela.

De acuerdo con las informaciones recogidas en el cuestionario, uno de los motivos más frecuentes fueron los que culturalmente marcaron la historia de exclusión de mujeres de los demás medios sociales. Una buena parte de las mujeres informó que no prosiguió sus estudios por cuestiones vinculadas al matrimonio, seguido de la gestación y demás contratiempos en la creación y cuidado de sus hijas o hijos pequeños y también porque "el esposo no dejaba estudiar". Esta situación también dejó fuera a muchas mujeres de las demás formas de participación social.

El número de mujeres que respondieron que trabajan "en el hogar, en la agricultura o reciben pensión o son jubiladas" corresponde a un grupo de mujeres que no asistieron a la escuela porque "vivían en el sitio". Muchas de ellas dejaron las zonas no urbanas para venir a vivir en las ciudades para acompañar a los hijos o hijas que alcanzaban la edad de escolarización que no se contemplaba en los locales donde vivían, o por motivo de fallecimiento del marido.

Estas familias eran mantenidas por el trabajo del"padre" que según las mujeres informaban, eran ellos quienes dictaban las reglas y de modo jerárquico controlaban cómo y cuándo las mujeres podían salir de casa. Las mujeres estudiantes justifican también que esta realidad ha cambiado mucho. Hoy son las principales mantenedoras de la casa junto a los hijos e hijas que ellas tuvieron que esperar crecer para poder volver a los estudios.

A pesar de estos cambios, lo que se planteó a través de los datos presentados en la Tabla 4, fue que aún el 35,44\% de las mujeres entrevistadas tiene el esposo como el principal mantenedor financiero, seguido del $29,16 \%$ de mujeres que son las principales responsables del sustento de la familia.

Tabla 4: Mantenimiento financiero de las mujeres entrevistadas

\begin{tabular}{lcc}
\hline Principal mantenedor financiero & TOTAL & $\%$ \\
\hline La pareja & 09 & 18,75 \\
El esposo & 17 & 35,44 \\
Ella misma & 14 & 29,16 \\
La pareja y los hijos/as & 02 & 4,16 \\
Ella misma y los hijos/as & 04 & 8,33 \\
Los padres o madres & 01 & 2,08 \\
Otras parientes (tía y hermana) & 01 & 2,08 \\
\hline TOTAL & 48 & 100 \\
\hline
\end{tabular}

Nota: Elaboración propia. 
Se evidenció que los motivos que dejaron a mujeres fuera de la escuela están relacionados con la criminalidad, el tráfico y el uso de drogas. Muchas estudiantes, preocupadas por quién y cómo dejar a sus hijos e hijas preadolescentes en casa solos para ir a estudiar la noche, optaron por abandonar el aula para evitar que los hijos y las hijas se involucraran con drogas u otra forma de práctica ilícita.

Mi mayor preocupación era dejar a sus hijos en casa. Ellos eran pequeños, adolescentes, y tenía mucho miedo que ellos se involucrar con drogas. Ahora ya están más grandes, es más fácil. Una hija ya estudia la noche. (Estudiante de la 2a serie de la EJA de la Escuela Joaquim Vicente Rondon, 39 años, información verbal).

Otras dejaron de estudiar, pues tuvieron muchas dificultades para criar solas a sus hijos e hijas, sin la participación del padre. Hoy el hijo está en el sistema penitenciario y ella puede volver a estudiar.

Porque entré en un mundo sin vuelta. Yo usé drogas y fui presa, y perdí a mis hijos por la justicia. Quisiera mucho ver de nuevo. Volví a estudiar para facilitar mi vida y mi situación con la justicia. Fui presa, perdí a mis hijos y mi casa. (Estudiante de la 3a serie de la EJA de la Escuela Nacional, 37 años, información verbal).

De acuerdo con nuestras entrevistadas, una de las mayores dificultades encontradas para permanecer en la escuela es la falta de seguridad pública, asociada con iluminación inadecuada y la falta de transporte público de calidad. Otra cuestión también encontrada es la relación con el trabajo y la escuela.

Vivir lejos y trabajar mucho y la calle es muy oscura y falta seguridad. Llegaba a casa muy amedrentada con la violencia que tenía en mi barrio. Ahora tiene escuela cerca. Mas antes, tenía que ir allá al centro para estudiar. (Estudiante del $4^{\circ}$ grado de la EJA de la Escuela Nacional, 46 años, información verbal).

Las estudiantes revelan que falta mucha seguridad en los barrios y que sería muy importante que en la noche la policía fuera más intensiva para proteger a la comunidad en el regreso de la escuela. En una de las entrevistas, se evidenció que por falta de seguridad adecuada en los barrios de Porto Velho, en una de las idas y venidas de la escuela fue violada, lo que ha tenido repercusiones con traumas a largo plazo.

He sido violada, abusada sexualmente, por eso fue a vivir en el interior donde no había escuela cercana. Tuve que trabajar durante mi época que era joven. De ahí pronto me casé y tuve que trabajar y cuidar el hijo pequeño para poder mantenerme. (Estudiante de la $3^{a}$ serie de la EJA de la Escuela Joaquim Vicente Rondon, 37 años, información verbal). 
doi: http://dx.doi.org/10.15359/ree.23-2.3

URL: http://www.una.ac.cr/educare

CORREO: educare@una.cr

La expectativa en el retorno a la escuela está íntimamente ligada a las cuestiones del tiempo y de la mejora en las condiciones financieras. En algunos momentos, cuestionadas en cuanto a qué hacer con lo que están aprendiendo en la escuela, estas mujeres plantean que están recuperando todo el "tiempo perdido" de forma que las hagan descubrir que el tiempo que quedaron fuera de la escuela tampoco se perdió.

Algunas mujeres informaron que lo que son hoy "no es más que lo que pasaron". Las dificultades que enfrentaron con la prohibición y las imposiciones del marido, el cuidado con los hijos e hijas, colocándolas como prioridades en ese momento, hacen que hoy ellas tengan más interés en permanecer en la escuela.

\section{Conclusiones}

Inicialmente, en esta investigación se constató la premisa del regreso de la mujer a la escolarización y que sus dificultades son diversas, pero que la fuerza de voluntad y autoestima están siempre presentes para levantarlas.

De las entrevistadas, en cuanto a las dificultades evidenciadas se puede tener en cuenta la falta de seguridad pública adecuada y la iluminación como un relevante aspecto de abandono de la escuela, seguido de otro factor importante que se planteó que consiste en la falta de apoyo de la familia. La falta de apoyo familiar para las participantes de la investigación tiene un valor de gran relevancia, pues la mayoría de las entrevistadas se refirió con énfasis a la importancia de la base familiar. Vale la pena resaltar que la mayoría de las estudiantes fueron madres precozmente, motivo que las alejó del aula, pues tuvieron que optar por cuidar y educar a su familia.

Otro aspecto fundamental es que la edad de estas estudiantes apuntó que de las 48 participantes 32 están por encima de 36 años. En cuanto al número de estudiantes de 46 a 65 años, este índice aún es mucho mayor. Para estas mujeres es interesante mostrar "que no importa la edad para iniciar o continuar los estudios", ellas quieren mostrar al mundo cuánto son capaces. En otras respuestas se percibió que la continuidad de los estudios es una realización personal, ya que pasaron parte sus vidas orientando a sus hijos e hijas en el estudio. También es interesante destacar que 17 mujeres se identificaron "del hogar" como su profesión, siendo que en la Tabla 4 la misma cuantidad de mujeres son sostenidas por el esposo.

Aunque los objetivos de la investigación han sido alcanzados, surge una serie de cuestionamientos que posibilitan nuevos estudios. Con los datos presentados en la Tabla 3, las categorías de estudiantes de 36 a 45 años y de 46 a 65 años, posibilita el surgimiento de un estudio específico para ese grupo de edad. Las participantes fueron colaboradoras importantes para que la problemática fuera respondida y fundamentada con el referencial teórico. Los resultados de esta investigación amplían la visión del retorno de las mujeres a la escuela con todas sus dificultades, donde la maternidad resulta un factor muy evidenciado en este estudio. 
El método utilizado para el estudio articuló la teoría presentada en la fundamentación teórica con las visitas a las cuatro escuelas participantes, y contribuyó en la configuración de los datos, principalmente en lo que se refiere a los factores socioeconómicos y culturales que retiran a las mujeres de la escuela para dedicarse exclusivamente al cuidado de sus pequeñas y pequeños, la maternidad y los quehaceres del hogar.

A partir de este estudio desarrollado en cuatro escuelas del municipio de Porto Velho, surgieron nuevas investigaciones relacionadas con la entrada al sistema educativo, en todos los Estados de Rondônia, o en regiones específicas del Estado, tanto en el tema de la formación profesional como en la enseñanza no superior. Otra propuesta para estudios futuros está relacionada con la categorización de mujeres que indica al profesorado como "del hogar", lo que sugiere un estudio específico sobre el retorno a la escuela pública del $35,41 \%$ o un estudio delimitado por mujeres entre 46 y 65 años.

Por lo tanto, las clases del primer segmento de la educación de jóvenes y personas adultas apuntaron un índice relevante de mujeres que regresan a la escuela con una perspectiva de ascenso escolar y profesional, así como en la participación efectiva en el presupuesto familiar, mejoría en la autoestima y en la calidad de vida.

\section{Referencias}

Andrade, S. dos S. (Agosto, 2008). Juventude, processos de escolarização e maternidade. In Seminário Internacional Fazendo Género, 8: Corpo, Violência e Poder (pp. 1-7). Florianópolis. Recuperado de http://www.fazendogenero.ufsc.br/8/sts/st58/sandra dos santos andrade 58.pdf

Congresso Nacional, Brasil. (20 de dezembro de 1996). Lei n. ${ }^{9}$ 9.394/96. Establece as Diretrizes e Bases da Educação Nacional. Recuperado de http://www.planalto.gov.br/ccivil 03/Leis/ L9394.htm.

Congresso Nacional, Brasil. (11 de agosto de 1971). Lei n. ${ }^{0}$ 5.692/71. Lei de Diretrizes e Bases para Ensino de $1 .^{\circ}$ e $2 .^{\circ}$ Graus. Recuperado de http://www.planalto.gov.br/ccivil 03/leis/L5692. htm.

Eiterer, C. L., Dias, J. D. y Coura, M. (2014). Aspectos da escolarização de mulheres na EJA. Perspectiva, 32(1), 161-180. doi: https://doi.org/10.5007/2175-795X.2014v32n1p161

Friedrich, M., Benite, A. M., Benite, C. R. M. y Pereira, V. S. (2010). Trajetória da escolarização de jovens e adultos no Brasil: De plataformas de governo a propostas pedagógicas esvaziadas. Ensaio: Avaliação e Políticas Públicas em Educação, 18(67), 389-410. doi: https:// doi.org/10.1590/S0104-40362010000200011 
doi: http://dx.doi.org/10.15359/ree.23-2.3

URL: http://www.una.ac.cr/educare

CORREO: educare@una.cr

Günther, H. (2006). Pesquisa qualitativa versus pesquisa quantitativa: Esta é a questão? Psicologia: Teoria e Pesquisa, 22(2), 201-209. doi: https://doi.org/10.1590/\$0102-37722006000200010

Moura, M. G. C. (2003). Educação de jovens e adultos: Um olhar sobre sua trajetória históricaa. Curitiba: Educarte.

Oro, A. C., Weschenfelder, R. C. S. y Stecanela, N. (2010). Mulheres e EJA: o que elas buscam? Rio Grande do Sul. Recuperado de https://docslide.com.br/documents/artigo-mulheres-eeja-o-que-elas-buscam.html

Siqueira, A. B. (2009). O retorno de jovens e adultos aos estudos formais após 20, 30, 40 anos. Poiésis, 2(3), 1-12. doi: https://doi.org/10.19177/prppge.v2e3200932-43

Schwartz, S. (2012). Alfabetización de jóvenes y adultos: Teoría y práctica. Petrópolis: Vozes.

Strelhow, T. B. (2010). Breve historia sobre a edução de jovens e adultos no Brasil. Revista Histedbr, 10(38), 49-59. Recuperado de http://www.histedbr.fe.unicamp.br/revista/edicoes/38/ art05 38.pdf 